\title{
PEG1/MEST and IGF2 DNA methylation in CIN and in cervical cancer
}

\author{
A. C. Vidal $\cdot$ N. M. Henry $\cdot$ S. K. Murphy $\cdot$ O. Oneko $\cdot$ M. Nye $\cdot$ \\ J. A. Bartlett $\cdot$ F. Overcash $\cdot$ Z. Huang $\cdot$ F. Wang $\cdot$ P. Mlay $\cdot$ J. Obure $\cdot$ \\ J. Smith · B. Vasquez $\cdot$ B. Swai $\cdot$ B. Hernandez $\cdot$ C. Hoyo
}

Received: 30 April 2013/ Accepted: 4 June 2013/Published online: 18 June 2013

(C) The Author(s) 2013. This article is published with open access at Springerlink.com

\begin{abstract}
Introduction Although most invasive cervical cancer (ICC) harbor $<20$ human papillomavirus (HPV) genotypes, use of HPV screening to predict ICC from HPV has low specificity, resulting in multiple and costly follow-up visits and overtreatment. We examined DNA methylation at regulatory regions of imprinted genes in relation to ICC and its precursor lesions to determine if methylation profiles are associated with progression of HPV-positive lesions to ICC. Materials and methods We enrolled 148 controls, 38 CIN and 48 ICC cases at Kilimanjaro Christian Medical Centre from 2008 to 2009. HPV was genotyped by linear array and HIV-1 serostatus was tested by two rapid HIV tests. DNA
\end{abstract}

A. C. Vidal $(\bowtie) \cdot$ N. M. Henry · F. Overcash · C. Hoyo

Department of Obstetrics and Gynecology, Program of Cancer Detection, Prevention and Control, Duke University School of Medicine, P.O. Box 104006, Durham, NC 27710, USA

e-mail: adriana.vidal@duke.edu

C. Hoyo

e-mail: hoyo0001@mc.duke.edu

S. K. Murphy $\cdot$ M. Nye $\cdot$ Z. Huang

Division of Gynecologic Oncology, Department of Obstetrics and Gynecology, Duke University School of Medicine,

Durham, NC 27708, USA

O. Oneko · P. Mlay · J. Obure

Department of Obstetrics and Gynecology, Kilimanjaro

Christian Medical Centre, Tumaini University, Moshi, Tanzania

\section{J. A. Bartlett}

Division of Infectious Diseases, Department of Medicine and Duke Global Health Institute, Duke University School of Medicine, Durham, NC 27710, USA

F. Wang

Duke Comprehensive Cancer Center, Duke University

Medical Center, Durham, NC 27710, USA methylation was measured by bisulfite pyrosequencing at regions regulating eight imprinted domains. Logistic regression models were used to estimate odd ratios.

Results After adjusting for age, HPV infection, parity, hormonal contraceptive use, and HIV-1 serostatus, a $10 \%$ decrease in methylation levels at an intragenic region of IGF2 was associated with higher risk of ICC (OR 2.00, $95 \%$ CI 1.14-3.44) and cervical intraepithelial neoplasia (CIN) (OR 1.51, $95 \%$ CI 1.00-2.50). Methylation levels at the H19 DMR and PEG1/MEST were also associated with ICC risk (OR 1.51, $95 \%$ CI 0.90-2.53, and OR 1.44, $95 \%$ CI 0.90-2.35, respectively). Restricting analyses to women $>30$ years further strengthened these associations.

\author{
J. Smith \\ Department of Epidemiology, University of North Carolina \\ at Chapel Hill, Chapel Hill, NC 27599, USA \\ B. Vasquez \\ Kilimanjaro Christian Medical Centre, Tumaini University, \\ Moshi, Tanzania \\ B. Vasquez \\ Tanzania and Duke Women's Health Collaboration, Durham, \\ NC 27710, USA \\ B. Swai \\ Department of Pathology, Kilimanjaro Christian Medical Centre, \\ Tumaini University, Moshi, Tanzania \\ B. Hernandez \\ Cancer Research Center of Hawaii, University of Hawaii, \\ Honolulu, HI 96822, USA
}


Conclusions While the small sample size limits inference, these findings show that altered DNA methylation at imprinted domains including IGF2/H19 and PEGI/MEST may mediate the association between HPV and ICC risk.

Keywords Insulin-like growth factor 2 gene (IGF2) . Paternally expressed gene $1 /$ mesoderm-specific transcript (PEGI/MEST) - Human papillomavirus (HPV) - Cervical intraepithelial neoplasia (CIN) - Invasive cervical cancer (ICC) · Differentially methylated regions (DMRs)

\section{Introduction}

Invasive cervical cancer (ICC) is the second most common cancer in women worldwide, with the heaviest burden borne by women in developing countries [1]. In the USA, more than 12,000 new ICC cases and 4,290 ICCrelated deaths were reported in 2010 [2]. Incidence was $60 \%$ higher and mortality was more than two times higher in Hispanics and African Americans compared with whites [1], despite comparable screening prevalence across racial/ethnic subgroups and comparable prevalence of infection with high-risk human papillomavirus (HRHPV) [3]. Current screening guidelines recommend liquid-based cytology screening methods followed by testing for HR-HPV genotypes, since most ICCs evolve slowly and harbor HR-HPV genotypes [4]. However, the sensitivity and specificity of existing screening tests remains suboptimal, leading to false positives and overtreatment which have been linked to subfertility and cervical stenosis [5]. Molecular features that together with known environmental factors can improve prediction of progression of cervical intraepithelial neoplasia (CIN) to ICC, are required.

DNA methylation has been extensively investigated in human cancers and other disorders with the aim of improving present diagnostic tools [6], but appropriate target sequences are unknown. Predictions based on genome-wide approaches have been proposed as reviewed by Wentzensen et al. [7]; however, findings have been difficult to replicate or interpret, because DNA methylation patterns are cell-type specific and may be transient [7]. DNA sequences at differentially methylated regions (DMRs) that regulate imprinted genes (a group of genes that are defined by expression from only a single parental allele) offer key advantages. DNA methylation profiles that regulate these genes are normally stably maintained throughout somatic cell division [8]. In cancer, imprinted DMRs are often deregulated, showing gains or losses in methylation that affect changes in expression and sometimes imprint status [9]. DNA methylation changes can result in either silencing of the only active allele or, alternatively, aberrant activation of the normally silenced allele, which may double the gene dosage [10]. Given that about $80 \%$ of known imprinted genes are found in clusters [8], altered methylation at a DMR can have wide-reaching effects within a cluster of genes.

The most intensively studied genomically imprinted gene, Insulin-like Growth Factor 2 (IGF2), encodes for IGF2, a potent mitogenic growth factor, the expression of which is controlled by DMRs located in the IGF2/H19 locus at chromosome $11 \mathrm{p} 15.5$. IGF2 is maternally imprinted and expressed from the paternal allele, while the $H 19$ gene is maternally expressed. At the $I G F 2 / H 19$ locus, aberrant methylation at DMRs that regulate the expression of $I G F 2$ have been associated with $I G F 2$ deregulation in many cancers, including colorectal, pancreatic and ovarian cancers and Wilms' tumors $[11,13]$. We have also identified a novel consensus binding site for the zinc finger protein, CTCF, located in IGF2 intron 3, and confirmed binding of CTCF to this region. The consensus sequence contains a single $\mathrm{CpG}$ dinucleotide whose methylation is strongly positively correlated with expression levels of $I G F 2$ in ovarian cancer (Huang and Murphy, in press). In addition, Paternally Expressed Gene 1/Mesoderm-Specific Transcript (PEGI/MEST) is an imprinted gene located at chromosome $7 \mathrm{q} 32.2$ and is expressed from the paternal allele. PEG1/MEST encodes for an enzyme belonging to the $\alpha / \beta$-hydrolase fold family [14]. The gene promoter of the active paternal allele of PEGI/MEST is hypomethylated while the transcriptionally silent maternal allele is hypermethylated, regulating the monoalleleic expression of PEGI/MEST. Deregulated methylation and/or expression of PEG1/MEST have been associated with invasive breast cancer [15] and uterine leiomyoma [16] in humans, and growth retardation, defective nurturing and increased lethality in mice [17].

We examine whether aberrant DNA methylation of the PEGI/MEST and IGF2/H19 regulatory regions and of three other imprinted gene DMRs belonging to this imprinted gene cluster: Pleomorphic Adenoma Gene-Like 1 (PLAGL1), Neuronatin (NNAT), Delta-like homolog 1 and noncoding Maternally Expressed Gene 3 (DLK1/MEG3), is associated with CIN and ICC, taking into account HPV infection.

\section{Materials and methods}

\section{Study participants}

Patient identification and enrollment procedures have been previously described [18, 19]. Briefly, between November 2008 and March 2009, eligible study participants $(n=249)$ were identified from the appointment books of 
the Reproductive Health Clinic (RHC) at KCMC, and the final dataset ( $86 \%$ of eligible participants or $n=234$ ) were women for whom questionnaires, CIN status, and HPV genotype data were available. The study was a collaboration between Duke University and the Reproductive Health Center at Kilimanjaro Christian Medical Centre (KCMC) in northern Tanzania, a cervical cancer prevention clinic funded by the World Health Organization (http://www.afro.who.int/en/tanzania/who-country-officetanzania.html).

Study procedures were approved by Research Ethics Boards at KCMC and Duke University.

Data collection

\section{Questionnaires}

A trained nurse-interviewer obtained informed consent from all participants and administered a 40-min in-person standardized questionnaire focusing on demographic information and gynecologic history.

\section{Specimens}

Two cervical scrapes were obtained from each participant using an Ayres spatula and Cytobrush and rinsed into Preserv-CytTM media (Hologic, Inc., Malborough, MA), as described [19].

\section{Ascertainment of CIN and carcinoma}

Pap smears and biopsy specimens were processed and read by the KCMC staff pathologist, using standard conventions and ASCCP guidelines as appropriate (http://www.asccp. org/ConsensusGuidelines/tabid/7436/Default.aspx). Cases were classified using the Bethesda classification system [20]. These clinical results were compiled and transferred securely to Duke University.

\section{HPV genotyping}

Purified DNA was obtained from ThinPrep ${ }^{\circledR}$ specimens and homogenized aliquotted biopsies and shipped to the University of Hawaii Cancer Center. PGMY09/PGMY11 primers [21] were used in PCR to target a 450-bp region of the HPV L1 gene, as described [19].

\section{Ascertainment of HIV-1 infection status}

Peripheral blood samples were centrifuged to separate the plasma and buffy coat. Plasma samples were used to test for HIV-1 infection using two rapid methods (Capillus HIV-1/HIV-2, Trinity Biotech PLC, Bray, County
Wicklow, Ireland, and Determine HIV-1/2, Abbott Laboratories, Abbott Park, IL). Reactive specimens were then tested using Western blot according to standard clinical practice (Genetic Systems HIV-1 Western blot kit, BioRad, Hercules, CA) [22].

\section{DNA methylation analyses}

Genomic DNA was prepared from cells isolated from the cervical scrapes or from biopsy specimens using PureGene protocol reagents (Qiagen; Valencia, CA). Genomic DNA was then modified by treatment with sodium bisulfite using the Zymo Easy-96 DNA Methylation Kit (Zymo Research, Irvine, CA). Bisulfite treatment of denatured DNA converts unmethylated cytosines to uracils but leaves methylated cytosines unchanged. When amplicons are sequenced, cytosines from methylated $\mathrm{CpG}$ sites appear as cytosine, while unmethylated cytosines appear as thymine. Pyrosequencing was performed using a Qiagen Pyromark Q96 MD Pyrosequencing instrument (Qiagen, Valencia, CA). The primer sequences and pyrosequencing assay conditions for the PEGI/MEST DMR, PLAGL1 DMR, MEG3 DMR, NNAT DMR, IGF2 DMR, and H19 DMR and assay validation using defined mixtures of methylated and unmethylated bisulfite-modified genomic DNA are described in detail elsewhere [23]. Methylation data were $98 \%$ complete.

The intragenic CTCF site was identified based on the $5^{\prime}$ CCGCNNGGNGNC- $3^{\prime}$ consensus previously defined [24] and is located in intron 3 of NCBI RefSeq NM_001127598. Binding of CTCF to this region was confirmed in ovarian cancer cells using chromatin immunoprecipitation as described (Huang and Murphy, in press). For quantitative methylation analysis of the intragenic region of $I G F 2$, we amplified bisulfite-modified DNAs using forward primer 5'-TTG GGA AGT TTT TGT TTG TTA GT-3' ${ }^{\prime}$ and biotinlabeled reverse primer $5^{\prime}$-ACC CTC AAA CCA AAC CCT AA- $3^{\prime}$ in a $25-\mu 1$ reaction mixture containing $40 \mathrm{ng}$ of bisulfite-modified template DNA, $1.5 \mathrm{mM} \mathrm{MgCl}_{2}$ and $0.12 \mu \mathrm{M}$ of each primer using the PyroMark PCR Kit (Qiagen, Valencia, CA). The sequence analyzed includes two $\mathrm{CpG}$ dinucleotides: one located within the consensus CTCF site and a second CpG outside the consensus motif ( $5^{\prime} \ldots$...gacc CGcagggtggctgCGtcc.... $3^{\prime} ;$ consensus underlined). We validated assay performance for linearity and the ability to detect increasing amounts of methylation across the full range of the assay (0-100 \% methylation) using defined mixtures of unmethylated and methylated bisulfite-modified genomic DNA (Epitect Bisulfite Controls, Qiagen). The methylation measured by pyrosequencing was highly correlated with the amount of methylated template included in these reactions $\left(R^{2}=0.99 ; p<0.0001\right)$. 


\section{IGF2 expression analysis}

Total RNA was extracted from 21 flash frozen ICC biopsy specimens using RNA Stat-60 (AMS Biotechnology, Abingdon, UK). cDNA was synthesized from $2 \mu \mathrm{g}$ total RNA by oligo-dT priming using SuperScript II Reverse Transcriptase (Invitrogen, Carlsbad, CA), as described [19].

\section{Statistical analyses}

Demographic and gynecological variables included age, history of pregnancy, number of pregnancies with complications (collected as a continuous variable and later dichotomized), and history of hormonal contraceptive use (dichotomized based on responses to ever having used either oral contraceptives, an intrauterine contraceptive device, medroxyprogesterone acetate, or a levonorgestrel intrauterine system). Chi-square tests were used to compare controls to CIN and ICC.

We interrogated between 3 and $8 \mathrm{CpG}$ sites per DMR: 3 for IGF2, 4 for H19, 4 for MEST, 6 for PLAGL1, 4 for $M E G 3-I G, 3$ for NNAT, and 8 for MEG3. To determine if a single mean was representative of methylation fractions at the multiple $\mathrm{CG}$ dinucleotides within the region analyzed, principal components analysis (PCA) was used. Cronbach's alphas for these regions were 0.95-0.99, suggesting that methylation of the CGs was sufficiently correlated, allowing a single mean to be used.

Women were classified as having HR-HPV if they tested positive for at least one HR-HPV genotype, as defined by current FDA-approved HPV molecular tests. High-risk genotypes include $16,18,31,33,35,39,45,51,52,56,58$, 59, 66, and 68; LR genotypes are 6, 11, 26, 40, 42, 53, 55, $61,62,69,70,72,73,81,82,83$, and 84. Multinomial logistic regression models were then used to evaluate the association between CIN/ICC and changes in DMR methylation, and adjusting for age, HPV infection, history of pregnancy, HIV-1 status, and hormonal contraceptive use. The association of potential confounders with CIN and cancer status was also evaluated using Chi-square tests. All statistical analyses were conducted in SAS 9.1 (SAS Institute, Cary, NC).

\section{Results}

Table 1 summarizes the characteristics of the 234 study participants. In all, 148 women (63\%) were classified as controls (women with neither CIN nor ICC). The remaining 86 women (37\%) were classified as cases; of these, 48 women $(21 \%)$ had ICC, 17 (7\%) had either CIN2 or CIN3, and 21 (9\%) had CIN1. The median age was 45 years; women with no cervical abnormalities and those with CIN1 were younger [mean 40.3 years $(\mathrm{SD}=9.9)$ and 35.7 years $(\mathrm{SD}=12.2)$, respectively] than women with cervical carcinoma or high-grade CIN [mean 55.2 years $(\mathrm{SD}=12.3)$ and $44.7(\mathrm{SD}=9.8)$ years, respectively]. All women with ICC and CIN2/3 were parous versus $86 \%$ of women with CIN1 and $95 \%$ of controls. Hormonal contraceptive use was more frequently reported by CIN cases $(59-76 \%)$ and controls $(68 \%)$ than ICC cases $(40 \%)$.
Table 1 Description of the Tanzanian women study participants as characterized by average age, HPV status, pregnancy and hormonal contraceptive use histories

\footnotetext{
* Using Fisher's exact test
}

\begin{tabular}{|c|c|c|c|c|c|}
\hline Characteristics & $\begin{array}{l}\text { Control }(n=148) \\
n(\%)\end{array}$ & $\begin{array}{l}\text { CIN1 }(n=21) \\
n(\%)\end{array}$ & $\begin{array}{l}\text { CIN2/3 }(n=17) \\
n(\%)\end{array}$ & $\begin{array}{l}\text { Cancer }(n=48) \\
n(\%)\end{array}$ & $p$ value \\
\hline Age (mean, SD) & $40.3(9.9)$ & $35.7(12.2)$ & $44.7(9.8)$ & $55.2(12.3)$ & $<0.0001$ \\
\hline \multicolumn{6}{|c|}{ History of pregnancy } \\
\hline Yes & $135(94.8)$ & $18(85.7)$ & $17(100)$ & $48(100)$ & \multirow[t]{3}{*}{$0.043^{*}$} \\
\hline No & $12(9.2)$ & $3(14.3)$ & $0(0.0)$ & $0(0.0)$ & \\
\hline Missing & $1(3)$ & & & & \\
\hline \multicolumn{6}{|c|}{ Hormonal contraceptive use } \\
\hline Ever & $98(67.6)$ & $16(76.2)$ & $10(58.8)$ & 19 (39.6) & \multirow[t]{3}{*}{0.0029} \\
\hline Never & $47(32.4)$ & $5(23.8)$ & $7(41.2)$ & $29(60.4)$ & \\
\hline Missing & 3 & & & & \\
\hline \multicolumn{6}{|l|}{ Any HPV } \\
\hline At least $1 \mathrm{HPV}$ & $20(14.1)$ & $12(66.7)$ & $14(87.5)$ & $33(89.2)$ & \multirow[t]{3}{*}{$<0.0001$} \\
\hline No HPV & $122(85.9)$ & $6(33.3)$ & $2(12.5)$ & $4(10.8)$ & \\
\hline Missing & 6 & 3 & 1 & 11 & \\
\hline \multicolumn{6}{|l|}{ HIV-1 Infection } \\
\hline Seropositive & $30(20.3)$ & $12(57.1)$ & $12(70.6)$ & 7 (14.6) & \multirow[t]{2}{*}{$<0.0001$} \\
\hline Seronegative & $118(79.7)$ & $9(42.9)$ & $5(29.4)$ & $41(85.4)$ & \\
\hline
\end{tabular}


Almost all (89 \%) women with cancer were positive for at least one HPV genotype whereas only a small proportion of controls $(14 \%)$ had detectable HPV.

Table 2 summarizes odds ratios (ORs) and $95 \%$ confidence intervals for the associations between $I G F 2, I G F 2$ intron 3, H19, PEG1/MEST, MEG3, PLAGL1 and NNAT methylation and CIN or ICC. When compared with controls, a $10 \%$ decrease in methylation at the $I G F 2$ intron 3 region (methylation mean $=71.97, \mathrm{SD}=8.5$ ) was associated with a two-fold increase in ICC risk (OR 2.00, $95 \%$ CI 1.14-3.44), after adjusting for age, gravidity, HIV-1 status, HPV infection, and oral contraceptive use. The association between lower methylation at the IGF2 intron 3 region and ICC risk remained essentially unchanged (OR 1.88, $95 \%$ CI 1.10-3.33) when restricting analysis to women aged $30+$ years. A similar pattern of association, albeit weaker, was also observed between a $10 \%$ methylation decrease for the IGF2 intron 3 region and CIN (OR 1.51, $95 \%$ CI 1.0-2.50).

Higher DNA methylation levels at the PEG1/MEST DMR were also associated with ICC (OR 1.44, $95 \%$ CI 0.90-2.35), and restricting analysis to women 30+ years revealed a somewhat stronger association (OR 2.13, $95 \%$ CI 1.10-4.16). Associations between DNA methylation at the IGF2 or H19 DMRs and risk of ICC or its precursor lesions were in a similar direction albeit non-

Table 2 Adjusted ORs and $95 \%$ CI for the association between mean methylation of six imprinted gene DMRs and CIN (1-3) and ICC

\begin{tabular}{|c|c|c|}
\hline & \multicolumn{2}{|l|}{ ORs (95\% CI) } \\
\hline & CIN (1-3) $n=26$ & Cancer $n=33$ \\
\hline \multicolumn{3}{|c|}{$I G F 2 / H 19$ imprinted domain } \\
\hline $\begin{array}{l}I G F 2 \text { intron } 3 \text { CTCF3 } \\
\text { DMR methylation }\end{array}$ & $1.51(1.00-2.5)$ & $2.00(1.14-3.44)$ \\
\hline $\begin{array}{l}I G F-2 \text { DMR } \\
\text { methylation }^{\mathrm{a}}\end{array}$ & $1.11(0.70-1.76)$ & $1.00(0.63-1.61)$ \\
\hline H-19 DMR methylation ${ }^{\mathrm{a}}$ & $0.99(0.57-1.71)$ & $1.51(0.90-2.53)$ \\
\hline $\begin{array}{l}\text { PEG1/MEST DMR } \\
\text { methylation }^{\mathrm{a}}\end{array}$ & $1.21(0.80-1.84)$ & $1.44(0.90-2.35)$ \\
\hline $\begin{array}{l}\text { PLAGL1 DMR } \\
\text { methylation }^{\mathrm{a}}\end{array}$ & $1.06(0.68-1.64)$ & $1.01(0.65-1.56)$ \\
\hline $\begin{array}{l}\text { MEG3 DMR } \\
\text { methylation }^{\mathrm{a}}\end{array}$ & $0.88(0.64-1.21)$ & $1.02(0.70-1.48)$ \\
\hline $\begin{array}{l}\text { MEG3_IG DMR } \\
\text { methylation }^{\mathrm{a}}\end{array}$ & $0.73(0.42-1.27)$ & $0.76(0.46-1.26)$ \\
\hline $\begin{array}{l}\text { NNAT DMR } \\
\text { methylation }^{\mathrm{a}}\end{array}$ & $1.05(0.74-.147)$ & $0.94(0.63-1.39)$ \\
\hline HPV & $24.17(6.61-88.46)$ & $48.96(9.17-261.50)$ \\
\hline
\end{tabular}

Adjusted for age, ever being pregnant, HIV-1 status, HPV infection, and oral contraceptive use

a $10 \%$ unit DNA methylation. All women with cancer had a history of pregnancy
Table 3 Regression coefficients and standard errors (SE) for the association between HR-HPV and LR-HPV genotypes and DMR methylation

\begin{tabular}{lll}
\hline $\begin{array}{l}\text { Chromosomal } \\
\text { region and } \\
\text { CpG site }\end{array}$ & $\begin{array}{l}\text { High-risk HPV } \\
\beta \text {-coefficient, } \\
\text { SE, } p \text { value }\end{array}$ & $\begin{array}{l}\text { Low-risk HPV } \\
\beta \text {-coefficient, } \\
\text { SE, } p \text { value }\end{array}$ \\
\hline$I G F 2$ intron 3 CTCF3 & $-8.55,1.75,<0.0001$ & $-17.74,4.70,0.0002$ \\
$I G F 2$ & $-3.38,0.85,<0.0001$ & $-4.88,2.17,0.02$ \\
$H 19$ & $-0.04,0.51,0.93$ & $-2.16,1.27,0.10$ \\
$P E G 1 / M E S T$ & $1.75,0.78,0.02$ & $2.80,1.87,0.13$ \\
\hline
\end{tabular}

Adjusted for multiple $\mathrm{CpGs}$

No HPV infection served as the baseline category

significant. Restricting the analyses to women older than 30 years did not alter these findings. No associations between DNA methylation and ICC, or CIN, were found at PLAGL1, NNAT and MEG3 DMRs (Table 2). The direction and magnitude of these associations remained unchanged when we excluded CIN1 lesions.

To examine whether DNA methylation at the $I G F 2$ intron 3 region, and at the IGF2, H19 and PEGI/MEST DMRs may mediate the association between HPV infection and ICC, we ran mixed models-to allow for unconstrained model entry of individual CpGs at each DMR - to evaluate associations between DNA methylation levels at each of these four DMRs and HPV infection, in which no HPV infection served as the baseline category (Table 3). We found that both HR-HPV and LR-HPV infections were statistically significantly associated with decreased DNA methylation at the IGF2 intron 3 region $(\beta=-8.7$, $\mathrm{SE}=1.75, \quad p<0.0001 ; \quad \beta=-17.74, \mathrm{SE}=4.70, \quad p=$ 0.0002 , respectively) and at the $I G F 2$ DMR $(\beta=-3.38$, $\mathrm{SE}=0.85, p<0.0001 ; \beta=-4.88, \mathrm{SE}=2.17, p=0.02$, respectively). HR-HPV infection was associated with higher methylation at the PEGI/MEST DMR $(\beta=1.75$, $\mathrm{SE}=0.78, p=0.02$ ). No associations were found for the H19 DMR.

To determine the functional significance of the association between DMR methylation at regulatory sequences of $I G F 2$, we computed correlation coefficients for DNA methylation and $\log -I G F 2$ gene expression from cervical cancer biopsies. We found higher IGF2 expression in samples where aberrant DNA methylation at $I G F 2$ regulatory regions was observed, compared to normal IGF2 methylation at these same regions $(r=0.6, p=0.001)$ (data not shown).

\section{Discussion}

We examined the association between DNA methylation at sequences regulating the PEGI/MEST, IGF2/H19, 
PLAGL1, MEG3 and NNAT imprinted domains and the risk of ICC and its precancerous lesions. After adjusting for age, lifetime gravidity, HIV-1 status, HPV infection, and oral contraceptive use, we found significant associations between altered DNA methylation levels at the PEGI/ MEST and IGF2/H19 imprinted domains, and risk of CIN and ICC. Our findings are consistent with the hypothesis that DNA methylation at regulatory sequences of imprinted genes represents susceptibility loci for ICC and its precursor lesions [7]. If confirmed in a larger number of these regulatory regions, these findings also suggest that DNA methylation, at these and perhaps other loci, may be developed for inclusion in risk stratification to identify individuals likely to progress from CIN to ICC.

The age at diagnosis in our Tanzania-based study is comparable to that of women in the USA and other developed countries, where ICC diagnosis tends to be in the fourth or fifth decades of life (SEER Stat Fact Sheets: Cervix Uteri), although this differs by racial/ethnic subgroup. In the USA, the age of peak incidence is 35-44 years in whites but $65+$ in African Americans and Hispanics, despite minimal differences in Pap smear screening rates (whites $91.4 \%$, African Americans $89.7 \%$ ) [25]. Age has been shown to influence methylation marks and other regions such as gene promoters but not in DMRs regulating imprinted genes [26], regions previously thought to be resistant to the effects of aging [27]. Our finding of a stronger association in women aged $30+$ are consistent with the findings of others [28] and suggests that DNA methylation marks in regulatory sequences of imprinted genes such as PEGI/MEST and IGF2/H19 can be harnessed to improve prediction of CIN cases likely to progress in older women.

Although imprinted gene dysregulation has been shown to contribute to the development and progression of many human cancers [29], DNA methylation in DMRs regulating imprinted genes causally associated with altered expression of multiple genes within imprint gene clusters remains understudied. The most intensively studied imprinted gene, $I G F 2$, has not yet been examined in relation to HPV infection and CIN progression. In the present study, we measured IGF2 transcript levels in women with ICC and found elevated IGF2 levels in samples where decreased methylation at the $I G F 2$ regulatory region was observed. Other studies have reported elevated IGF2 levels among women with cervical cancer and high- but not low-grade squamous intraepithelial lesions when compared with women with no history of abnormal Pap tests. Bulky tumors $(>3 \mathrm{~cm})$ are more common in women with higher IGF2 protein levels than in those with smaller tumors $(<3 \mathrm{~cm})$ [30]. It is possible that aberrant methylation in some $\mathrm{CpGs}$ in the IGF2/H19 imprinted domain such as the intron $3 \mathrm{CTCF}$ site may be influencing loss of imprinting and the exclusive usage of $I G F 2$ promoter 1, inducing
IGF2 overexpression, as has been previously shown in cervical carcinomas [30]. Albeit IGF2 DMR aberrant methylation has been linked with other cancers [12, 13], this is the first time that $I G F 2$ intron 3 methylation has been examined in relation to cervical cancer.

Our findings of a strong association between HR-HPV infection and methylation levels support the hypothesis that aberrant DNA methylation may mediate the association between HPV infection and risk of CIN and ICC. If aberrant methylation occurs early in development, it would increase susceptibility to HPV infection and subsequently lesion progression, via elevated expression of these growth effectors - the IGF2 and PEG1/MEST genes.

The study's main limitation was its small sample size, which required combining CIN1-3 cases even though a sizable proportion of lower-grade CIN cases may not progress. A second limitation is that only a small fraction $(<4 \%)$ of Tanzanian women over 30 years of age are nulliparous (National Bureau of Statistics [Tanzania], ICF Macro. 2011), making parity-related sub-analyses difficult. Third, a larger sample size would enable testing the hypothesis of age-dependent DNA methylation in ICC. Finally, PEG1/MEST transcripts were not compared between normal and aberrant methylation as samples were limited; thus, the functional significance of aberrant DNA methylation can be inferred only from previous molecular data. However, the present data suggest that decreased methylation at the $I G F 2$ regulatory region upregulates IGF2 expression in cervical cancer tissue. Our study provides early empirical evidence for associations between DNA methylation patterns in regulatory sequences of imprinted gene and cervical cancer and its precursors. Despite these limitations, we found moderately strong associations between aberrant DNA methylation and ICC and its precursors, independent of known risk factors.

In summary, altered methylation at the PEG1/MEST DMR and at the IGF2 intron 3 CTCF site was associated with increased ICC risk and HR-HPV infection. Larger studies are required to confirm our findings.

Acknowledgments This paper was supported in part by grants from the National Institutes of Health K01 CA104517, R01CA142983, R01CA142983-02S1 and Duke CFAR Grant Number 5P30 AI 064518 .

Open Access This article is distributed under the terms of the Creative Commons Attribution License which permits any use, distribution, and reproduction in any medium, provided the original author(s) and the source are credited.

\section{References}

1. Bray F, Ren J-S, Masuyer E, Ferlay J. Global estimates of cancer prevalence for 27 sites in the adult population in 2008. Int J Cancer. 2012;132:1133-45. 
2. Saslow D, Solomon D, Lawson HW, Killackey M, Kulasingam SL, Cain J, et al. American Cancer Society, American Society for Colposcopy and Cervical Pathology, and American Society for Clinical Pathology screening guidelines for the prevention and early detection of cervical cancer. CA Cancer J Clin. 2012;137:516-42.

3. Kahn JA, Lan D, Kahn RS. Sociodemographic factors associated with high-risk human papillomavirus infection. Obstet Gynecol. 2007;110:87-95.

4. Walboomers JM, Jacobs MV, Manos MM, Bosh FX, Kummer JA, Shah KV, et al. Human papillomavirus is a necessary cause of invasive cervical cancer worldwide. J Pathol. 1999;189:12-9.

5. Schiffman M, Castle PE, Jeronimo J, Rodriguez AC, Wacholder S. Human papillomavirus and cervical cancer. Lancet. 2007;370:890-907.

6. Vucic EA, Brown CJ, Lam WL. Epigenetics of cancer progression. Pharmacogenomics. 2008;9:215-34

7. Wentzensen N, Sherman ME, Schiffman M, Wang SS. Utlity of methylation markers in cervical cancer early detection: appraisal of the state-of-the-science. Gynecol Oncol. 2009;112:293-9.

8. Reik W, Walter J. Genomic imprinting: parental influence on the genome. Nat Rev Genet. 2001;2:21-32.

9. Mai M, Qian C, Yokomizo A, Tindall DJ, Bostwick D, Polychronakos C, et al. Loss of imprinting and allele switching of p73 in renal cell carcinoma. Oncogene. 1998;17:1739-41.

10. Kerr NJ, Chun YH, Yun K, Hathcott RW, Reeve AE, Sullivan MJ. Pancreatoblastoma is associated with chromosome $11 \mathrm{p}$ loss of heterozygsity and IGF2 overexpression. Med Pediatr Oncol. 2002;39:52-4.

11. Cui H, Onyango P, Brandenburg S, Wu Y, Hsieh CL, Feinberg AP. Loss of imprinting in colorectal cancer linked to hypomethylation of H19 and IGF2. Cancer Res. 2002;62:6442-6.

12. Sullivan MJ, Taniguchi T, Jhee A, Kerr N, Reeve AE. Relaxation of IGF2 imprinting in Wilms tumours associated with specific changes in IGF2 methylation. Oncogene. 1999;18:7527-34.

13. Murphy SK, Huang Z, Wen Y, Spillman MA, Whitaker RS, Simel LR, et al. Frequent IGF2/H19 domain epigenetic alterations and elevated IGF2 expression in epithelial ovarian cancer. Mol Cancer Res. 2006;4:283-92.

14. Riesewijk AM, Hu L, Schulz U, Tariverdian G, Hoglund P, Kere J, et al. Monoallelic expression of human PEG1/MEST is paralleled by parent-specific methylation in fetuses. Genomics. 1997;42:236-44.

15. Pedersen IS, Dervan PA, Broderick D, Harrison M, Miller N, Delany E, et al. Frequent loss of imprinting of PEG1/MEST in invasive breast cancer. Cancer Res. 1999;59:5449-51.

16. Moon YS, Park SK, Kim HT, Lee TS, Kim JH, Choi YS. Imprinting and expression status of isoforms 1 and 2 of PEG1/MEST gene in uterine leiomyoma. Gynecol Obstet Invest. 2010;70:120-5.
17. Lefebvre L, Viville S, Barton SC, Ishino F, Keverne EB, Surani MA. Abnormal maternal behaviour and growth retardation associated with loss of the imprinted gene Mest. Nat Genet. 1998;20:163-9.

18. Vidal AC, Murphy SK, Hernandez BY, Vasquez B, Bartlett JA, Olola O, et al Distribution of HPV genotypes in cervical intraepitheliallesions and cervical cancer in Tanzanian women. Infect Agent Cancer. 2011;6:20.

19. Nye M, Hoyo C, Huang Z, Vidal AC, Wang F, Overcash F, et al. Associations between methylation of Paternally Expressed Gene 3, cervical intraepithelial neoplasia and invasive cervical cancer in Tanzania. PLoS One. 2013;8:e56325.

20. Apgar BS, Zoschnick L, Wright TC Jr. The 2001 Bethesda System terminology. Am Fam Phys. 2003;68:1992-8.

21. Gravitt PE, Kamath AM, Gaffikin L, Chirenje ZM, Womack S, Shah KV. Human papillomavirus genotype prevalence in high-grade squamous intraepithelial lesions and colposcopically normal women from Zimbabwe. Int J Cancer. 2002;100:729-32.

22. Mayhood MK, Afwamba IA, Odhiambo CO, Ndanu E, Thielman NM, Morrissey $\mathrm{AB}$, et al. Validation, performance under field conditions, and costeffectiveness of Capillus HIV-1/HIV-2 and determine HIV-1/2 rapid human immunodeficiency virus antibody assays using sequential and parallel testing algorithms in Tanzania. J Clin Microbiol. 2008;46:3946-51.

23. Murphy SK, Huang Z, Hoyo C. Differentially methylated regions of imprinted genes in prenatal, perinatal and postnatal tissues. PLoS One. 2012;7:e40924.

24. Rosa AL, Wu YQ, Kwabi-Addo B, Coveler KJ. Reid Sutton V, Shaffer LG. Allelespecific methylation of a functional CTCF binding site upstream of MEG3 in the human imprinted domain of 14q32. Chromosome Res. 2005;13:809-18.

25. Barnholtz-Sloan J, Patel N, Rollison D, Kortepeter K, MacKinnon J, Giuliano A. Incidence trends of invasive cervical cancer in the United States by combined race and ethnicity. Cancer Causes Control. 2009;20:1129-38.

26. Woodfine K, Huddleston JE, Murrell A. Quantitative analysis of DNA methylation at all human imprinted regions reveals preservation of epigenetic stability in adult somatic tissue. Epigenetics Chromatin. 2011;4:1.

27. Issa J-P, Vertino PM, Boehm CD, Newsham IF, Baylin SB. Switch from monoallelic to biallelic human IGF2 promoter methylation during aging and carcinogenesis. Proc Natl Acad Sci. 1996;93:11757-62.

28. Teschendorff AE, Menon U, Gentry-Maharaj A, Ramus SJ, Weisenberger DJ, Shen $\mathrm{H}$, et al. Age-dependent DNA methylation of genes that are suppressed in stem cells is a hallmark of cancer. Genome Res. 2010;20:440-6.

29. Momparler RL. Cancer epigenetics. Oncogene. 2003;22:6479-83.

30. Mathur SP, Mathur RS, Gray EA, Lane D, Underwwod PG, Kohler M, et al. Serum vascular endothelial growth factor C (VEGF-C) as a specific biomarker for advanced cervical cancer: relationship to insulin-like growth factor II (IGFII), IGF binding protein 3 (IGF-BP3) and VEGF-A [corrected]. Gynecol Oncol. 2005;98:467-83. 